\title{
BoS1
}

\section{SAGEEP1 Multi-elevation Calibration of Frequency Domain Electromagnetic Data}

\author{
B.J. Minsley* (U.S. Geological Survey), G. Hodges (Fugro Airborne), B.D. \\ Smith (U.S. Geological Survey) \& J.D. Abraham (U.S. Geological Survey)
}

\section{SUMMARY}

The ability to make quantitative inferences about subsurface properties is an important component of interpreting frequency domain electromagnetic (FDEM) data. Systematic data errors caused by imperfect instrument calibration can lead to inversion artifacts or, in some cases, best-fit models that are inconsistent with the measured data. Factory and inflight internal system calibrations have helped to reduce, though not always eliminate, calibration errors in modern FDEM systems. A number of methods have been developed to calibrate data after it has been acquired, but these are primarily based on having auxiliary information about subsurface properties from well logs or ground-based geophysical surveys, which are not always available and may have inaccuracies of their own.

In this work, we propose a new strategy for calibrating FDEM data that does not rely on prior knowledge of the subsurface structure. This calibration procedure involves acquiring multiple datasets along a single calibration line at several different survey elevations at the beginning of a survey. Calibration parameters, consisting of gain, phase, and bias correction factors for each frequency, are derived by requiring that data from the multiple survey elevations must be consistent with the same earth model at each location along the line. This is accomplished by simultaneously inverting the multi-elevation data for an earth model at each location along the profile along with a single set of calibration parameters. This joint inversion strategy recovers the combination of earth models and calibration parameters that are optimally consistent with the multi-elevation data. The derived calibration parameters are then applied to the survey data, and the calibration procedure can be repeated as necessary to correct for system drift. 
The ability to make quantitative inferences about subsurface properties is an important component of interpreting frequency domain electromagnetic (FDEM) data. Systematic data errors caused by imperfect instrument calibration can lead to inversion artifacts or, in some cases, best-fit models that are inconsistent with the measured data. Factory and inflight internal system calibrations have helped to reduce, though not always eliminate, calibration errors in modern FDEM systems. A number of methods have been developed to calibrate data after it has been acquired, but these are primarily based on having auxiliary information about subsurface properties from well logs or ground-based geophysical surveys, which are not always available and may have inaccuracies of their own.

In this work, we propose a new strategy for calibrating FDEM data that does not rely on prior knowledge of the subsurface structure. This calibration procedure involves acquiring multiple datasets along a single calibration line at several different survey elevations at the beginning of a survey. Calibration parameters, consisting of gain, phase, and bias correction factors for each frequency, are derived by requiring that data from the multiple survey elevations must be consistent with the same earth model at each location along the line. This is accomplished by simultaneously inverting the multi-elevation data for an earth model at each location along the profile along with a single set of calibration parameters. This joint inversion strategy recovers the combination of earth models and calibration parameters that are optimally consistent with the multi-elevation data. The derived calibration parameters are then applied to the survey data, and the calibration procedure can be repeated as necessary to correct for system drift. 